\title{
SPECTROSCOPIC MODELING OF THE ALGOL-TYPE STAR TW DRACONIS
}

\author{
A. TKachenko ${ }^{1}$, H. Lehmann ${ }^{1}$, and D. Mkrtichian ${ }^{2}$ \\ ${ }^{1}$ Thüringer Landessternwarte (TLS), D-7778 Tautenburg, Germany \\ ${ }^{2}$ Crimean Astrophysical Observatory, Nauchny, Crimea, 98409, Ukraine \\ Received 2009 September 4; accepted 2010 January 16; published 2010 February 19
}

\begin{abstract}
TW Dra is a well-known short-period Algol-type system. It belongs to the class of oEA stars (Algol-type systems with a $\delta$ Scuti-like oscillating primary component). We investigate the TW Dra system based on high-resolution spectra taken in 2007 and in 2008 to derive precise stellar and system parameters and to check for phases of active mass transfer. We derived a precise orbital solution and the extracted spectra of the stellar components of the TW Dra system. The analysis of the spectrum of the primary shows that it is a normal A5-type star with a chemical composition close to solar. By means of the Shellspec07_inverse program we calculated precise stellar and system parameters from the composite spectra. All results agree with those from the most recent photometric study. During both epochs of observations, the system can be well modeled without counting for mass transfer effects, assuming a spherical configuration of the primary and a Roche-lobe filling secondary. Thus, we conclude that the star was in quiet phases during both epochs of observations.
\end{abstract}

Key words: binaries: eclipsing - binaries: spectroscopic - stars: individual (TW Dra)

Online-only material: color figures

\section{INTRODUCTION}

TW Dra (spectral type A5 V+K0 III) is a bright Algol-type system with an orbital period of 2.807 days. It is in turn the bright component of the visual binary ADS 9706 . The primary eclipse is a total one (Popper 1989). A first photoelectric study of the light variation was made by Baglow (1952). Kopal \& Shapley (1956) estimated the masses of the components to $M_{1}=1.9 M_{\odot}$ and $M_{2}=0.82 M_{\odot}(q=0.43)$. Giuricin et al. (1980) analyzed the photoelectric data obtained by Baglow (1952) and Walter (1978) and favored the results based on the later data set. They found $q=0.47$ in good agreement with the value given by Popper (1989) based on the radial velocities. Al-Naimiy \& Al-Sikab (1984) analyzed the star's light curve and determined the radius of the primary and separation to be $2.5 R_{\odot}$ and 12 $R_{\odot}$, respectively. Their findings were in good agreement with those by Kopal \& Shapley (1956) and Giuricin et al. (1980). The most comprehensive study so far was conducted by M. Zejda et al. (2010, in preparation). Zejda et al. derived atmospheric and geometrical parameters based on radial velocities using the program FOTEL by Hadrava (2004a) and on light curve analysis using PHOEBE (Prša \& Zwitter 2005).

Kusakin et al. (2001) discovered short-term light variability with a semi-amplitude of 2 mmag in the primary component of TW Dra, detecting one frequency peak at $17.99 \mathrm{c} \mathrm{d}^{-1}$. Kim et al. (2003) photometrically investigated the system again and found a variability with $5 \mathrm{mmag}$ semi-amplitude at orbital phases near to secondary minimum. The derived oscillation frequency of $18.95 \mathrm{c} \mathrm{d}^{-1}$ is the $1 \mathrm{c} \mathrm{d}^{-1}$ alias of the value given by Kusakin et al. (2001). Authors also found a second frequency peak near to $27 \mathrm{c} \mathrm{d}^{-1}$ suggesting that TW Dra is a multi-periodic pulsator. The first dominating pulsation mode was also found by Zejda et al. (2006) in the vicinity of the primary minimum.

Lehmann et al. $(2008,2009)$ detected three oscillation modes of 22.90, 14.06, and $24.72 \mathrm{c} \mathrm{d}^{-1}$ from the line profile variations having semi-amplitudes of $0.3 \mathrm{~km} \mathrm{~s}^{-1}$. They could not find the $17.99 \mathrm{c} \mathrm{d}^{-1}$ mode detected by Kusakin et al. (2001), however. The authors tried to identify the modes and could limit the range in $l$ and $m$ to 7-12. They suggested that these high-degree modes are most likely sectoral modes but no unique identification could be derived.

TW Dra has long been known to show orbital period variations. A historical summary about this topic is given in Zejda et al. (2008). Qian \& Boonrucksar (2002) found a secular increase of the orbital period by $4.43 \times 10^{-6}$ days $\mathrm{yr}^{-1}$ that they attributed to a dynamical mass transfer from the secondary to the primary with a rate of $6.8 \times 10^{-7} M_{\odot} \mathrm{yr}^{-1}$. Besides this secular change, the authors report on two irregular period jumps (increases with successive decreases) which they explain by structure variations of the cool giant star, possibly related to its magnetic activity cycle. Zejda et al. (2008) inspected the system for orbital period changes again. They found that TW Dra showed an almost constant orbital period during the first observations in 1858-1905 whereas in 1905-1942 the period increased corresponding to a mass exchange rate of $3.9 \times 10^{-7}$ $M_{\odot} \mathrm{yr}^{-1}$. After that period of mass transfer, the star showed alternating cycles of increasing and decreasing orbital period that could be related to the magnetic activity cycle of the cool giant star. These period changes are overlaid by other cyclic variations of much smaller amplitude caused by the light time effect of a third body with a period of 6.5 years.

In our present work, we investigate the TW Dra system using several methods. We use the KOREL program (Hadrava 2004b) to compute the orbital solution (Section 4) and the extracted spectra of the components (Section 5) of the Algol-type system. The derived orbital period and time of primary minimum are used to build the orbital phase binned spectra needed by Shellspec07_inverse for further analysis. The extracted spectra are analyzed with the SynthV program (Tsymbal 1996) based on atmosphere models calculated with the LLmodels code (Shulyak et al. 2004; Section 6). Finally, we apply the Shellspec07_inverse program that was designed for the finetuning of stellar and system parameters of eclipsing binaries from spectroscopic data to the spectra of TW Dra taken in 2007 and 2008 (Section 7). TW Dra is the second Algol-type system that we investigate using Shellspec07_inverse. Its first application, to the oscillating Algol-type star RZ Cas, is described in detail in Tkachenko et al. (2009). 
Table 1

Journal of Observations ${ }^{\text {a }}$

\begin{tabular}{|c|c|c|c|c|c|c|c|c|c|c|c|}
\hline Date & JD & $N$ & Source & Date & JD & $N$ & Source & Date & JD & $N$ & Source \\
\hline 2007 April 24 & 215 & 17 & TLS & 2007 May 07 & 228 & 42 & BOAO & 2008 March 28 & 554 & 15 & TLS \\
\hline 2007 April 25 & 216 & 20 & TLS & 2007 May 08 & 229 & 19 & BOAO & 2008 March 29 & 555 & 42 & TLS \\
\hline 2007 April 26 & 217 & 32 & TLS & 2007 May 10 & 231 & 9 & BOAO & 2008 March 30 & 556 & 12 & TLS \\
\hline 2007 April 27 & 218 & 20 & TLS & 2008 March 17 & 543 & 2 & TLS & 2008 April 16 & 573 & 43 & TLS \\
\hline 2007 April 28 & 219 & 14 & TLS & 2008 March 18 & 544 & 2 & TLS & 2008 April 17 & 574 & 24 & TLS \\
\hline 2007 April 29 & 220 & 11 & TLS & 2008 March 21 & 547 & 3 & TLS & 2008 April 18 & 575 & 3 & TLS \\
\hline 2007 April 30 & 221 & 13 & TLS & 2008 March 23 & 549 & 3 & TLS & 2008 April 20 & 577 & 28 & TLS \\
\hline 2007 May 01 & 222 & 33 & TLS & 2008 March 24 & 550 & 40 & BOAO & 2008 April 22 & 579 & 1 & TLS \\
\hline 2007 May 02 & 223 & 49 & TLS & 2008 March 24 & 550 & 1 & TLS & 2008 April 23 & 580 & 40 & TLS \\
\hline 2007 May 03 & 224 & 40 & TLS & 2008 March 25 & 551 & 2 & TLS & 2008 April 24 & 581 & 17 & TLS \\
\hline 2007 May 04 & 225 & 30 & TLS & 2008 March 26 & 552 & 69 & $\mathrm{BOAO}$ & 2008 April 25 & 582 & 24 & TLS \\
\hline 2007 May 05 & 226 & 29 & TLS & 2008 March 27 & 553 & 19 & BOAO & 2008 April 26 & 583 & 40 & TLS \\
\hline 2007 May 06 & 227 & 18 & TLS & 2008 March 27 & 553 & 12 & TLS & 2008 April 27 & 584 & 37 & TLS \\
\hline
\end{tabular}

Note. ${ }^{\text {a }}$ JD is the Julian Date $2454000+, N$ is the number of spectra obtained in single nights.

\section{OBSERVATIONS}

Three hundred and ninety-six spectra of TW Dra were obtained in 2007 April/May and 479 spectra in 2008 March/ April. Spectra were taken with the coudé echelle spectrograph at the $2 \mathrm{~m}$ telescope of the Thüringer Landessternwarte Tautenburg (TLS) and with the BOES spectrograph at the $1.8 \mathrm{~m}$ telescope of the Bohyunsan Optical Astronomy Observatory (BOAO). TLS spectra have a spectral resolution of 33,000 and cover a wavelength range from $4700 \AA$ A to $7400 \AA$. BOES spectra have a resolution of 50,000 and cover a larger wavelength range down to $3600 \AA$. The typical exposure time was 10 minutes, and the spectra have a typical signal-to-noise ratio $(\mathrm{S} / \mathrm{N})$ of 100 . Table 1 gives the journal of the observations. The most extended period of continuous observations without gaps comprises 15 consecutive nights in 2007.

Spectra have been reduced using standard MIDAS packages. Reduction included bias and stray light subtraction, filtering of cosmic rays, flat fielding, optimum extraction of echelle orders, wavelength calibration using a Th-Ar lamp, correction for instrumental shifts using a large number of telluric lines, normalization to local continuum, and weighted merging of orders.

\section{LIGHT FROM THE THIRD COMPONENT}

TW Dra is the bright component of the visual binary ADS 9706. It consists of the unresolved Algol-type system (components A and B) and a third component in a distance of 3 arcsec. Besides light from the two components of the Algol-type system, spectra partly include light from the third component, depending on the slit orientation of the spectrograph (the field of view of the coudé spectrograph rotates with the hour angle) and on the seeing conditions during the observations (typical seeing of 2 arcsec). Figure 1 shows the time series of line profiles obtained in 2007 folded with the orbital period. The horizontal axis spans $\pm 300 \mathrm{~km} \mathrm{~s}^{-1}$, the vertical axis gives the orbital phase from -0.25 (bottom) to +0.75 (top). Line profiles were built by co-adding the nine most unblended metal lines: Fe I 4958 (narrow doublet), 5456 (narrow doublet), 5616, 5625, 6065, and $6192 \AA$, Ca I $6122 \AA$, and Si II 6371, $6347 \AA$ on the radial velocity (RV) scale followed by orbital phase binning. From Figure 1, we clearly see the varying contribution of the third component in form of the straight and sharp vertical lines that show no visible RV variation during the period of observations. The Rossiter-McLaughlin effect can be seen as the S-shaped dark feature during primary eclipse (phase zero)

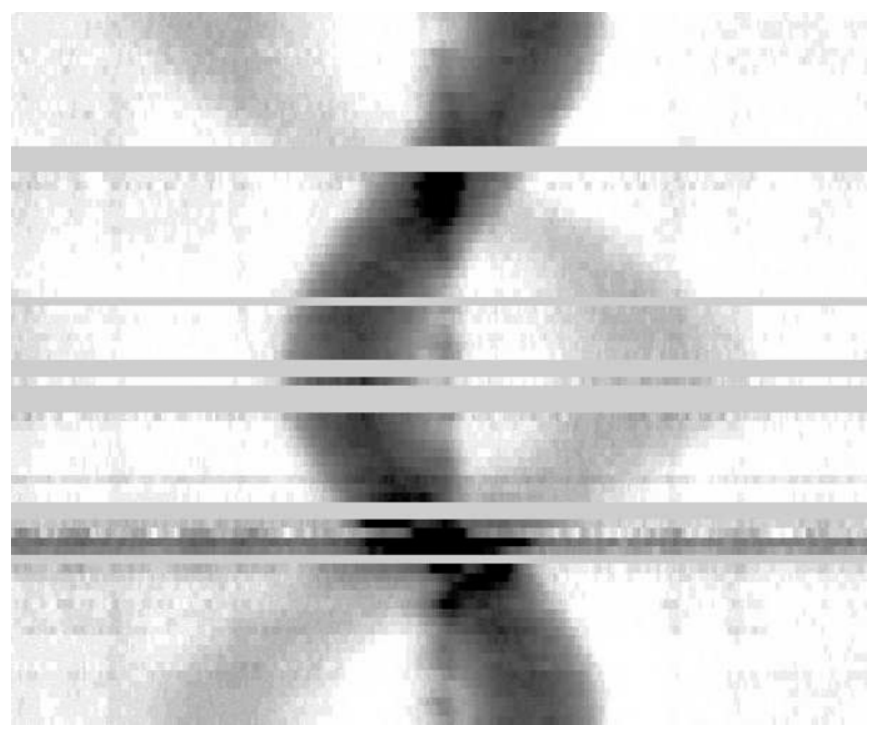

Figure 1. Time series of co-added line profiles folded with the orbital period.

and less pronounced during secondary eclipse. Horizontal gray lines are due to the incomplete phase coverage.

The unpredictable contribution of the third component causes serious complications in the line profile analysis. Figure 2 shows one of the profiles. Here, the contribution of the third component can be seen as the distortion in the blue wing of the deep line of the primary. A fit by three Gaussians gives only a rough approximation and works only at orbital phases of larger separation.

\section{ORBITAL SOLUTION}

We used KOREL (Hadrava 2004b) to obtain the orbital solution and the extracted spectra of the three components using the wavelength range $4895-5670 \AA$ that is almost free of telluric lines. Separate KOREL solutions for years 2007 and 2008 showed that the obtained periods and epochs of minimum do not differ significantly from each other. Assuming that the values derived from the times of minima will be more precise than attainable from the time sampling of our spectra, we fixed the epoch of Min I and the orbital period to the values computed from Zejda's formulae (Zejda et al. 2008) for the middle of all our observations: Min I = 2454 400.97997, $P=2.8068491$ days. 


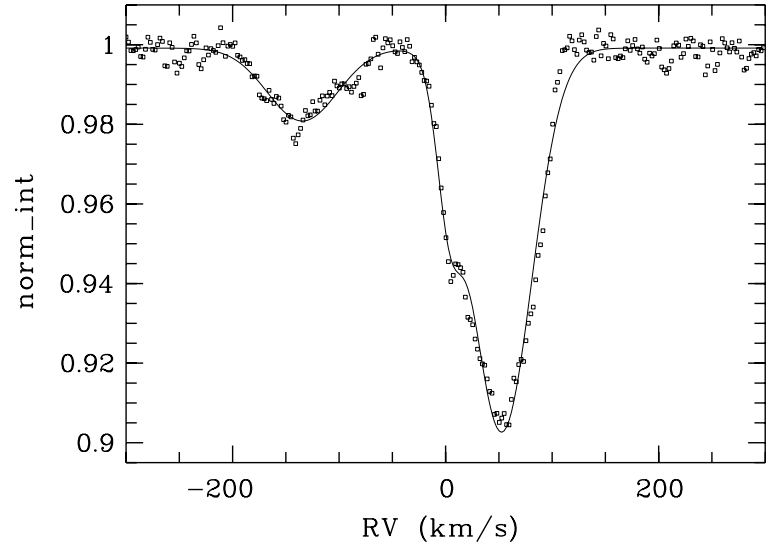

Figure 2. Fit of one composite line profile by three Gaussians (example).

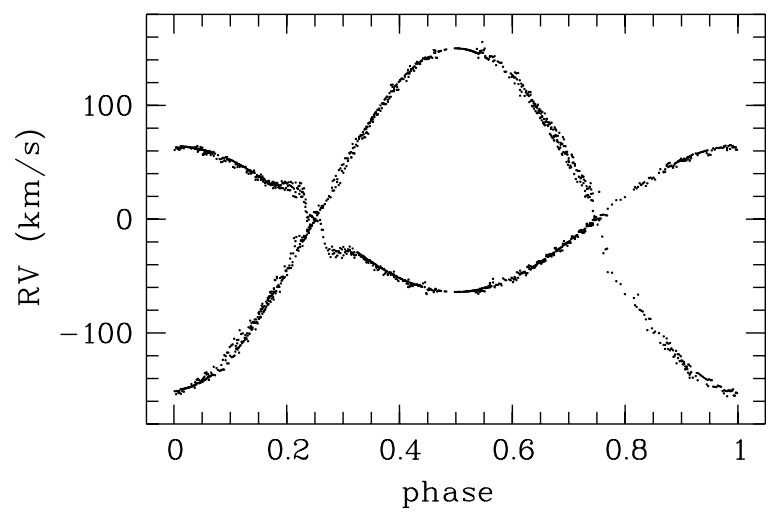

Figure 3. Radial velocities of primary and secondary obtained with KOREL, folded with the orbital period.

Table 2

Orbital Elements Derived with KOREL

\begin{tabular}{lccc}
\hline \hline \multicolumn{1}{c}{ Element } & Primary & Secondary & Third \\
\hline$P(\mathrm{~d})$ & \multicolumn{2}{c}{2.8068491 (fixed) } & \\
$T$ & 2454400.97997 (fixed) & \\
$K_{1}\left(\mathrm{~km} \mathrm{~s}^{-1}\right)$ & $64.05(34)$ & $150(2.3)$ & \\
$\gamma\left(\mathrm{km} \mathrm{s}^{-1}\right)$ & $0.68(71)$ & $0.7(1.1)$ & $1.93(41)$ \\
$q$ & \multicolumn{2}{c}{$0.427(11)$} & \\
\hline
\end{tabular}

The orbital elements derived with KOREL are listed in Table 2 where $T$ gives the time of Min I. Errors in units of the last digits are given in parentheses. The computed RVs are shown in Figure 3. For a better visualization, phase zero corresponds in Figures 3-5 to the orbital phase of largest RV separation. Figure 4 shows the deviations of these RVs from the calculated orbital solution. Note that this deviation is identical to zero for the majority of data points which means that in most cases KOREL shifted the corresponding spectra by exactly the value of the calculated orbital RV to build the extracted spectra of the components. For this reason, it is not possible to use the RVs computed by KOREL for an error estimation of the calculated elements. The errors of $K_{1}$ and $q$ as listed in Table 2 have been estimated from the $\chi^{2}$-distribution obtained from running KOREL with fixed input parameters on a twodimensional grid in $K_{1}$ and $q$. Absolute values of the $\gamma$-velocity have been measured from the extracted spectra by comparing the positions of a large number of metal lines with the corresponding laboratory wavelengths.

From Figure 4 it can be seen that there is no systematic difference between the data from 2007 and 2008. We see a

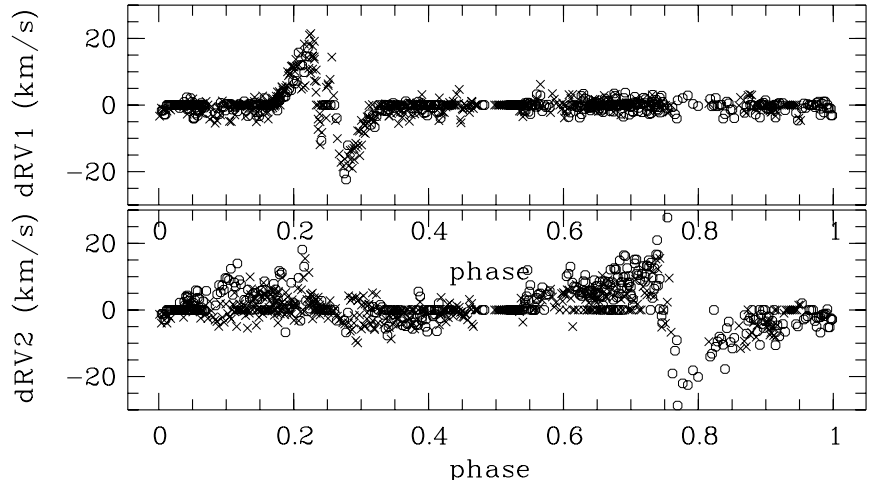

Figure 4. Same as Figure 3 but for the deviation of KOREL RVs from the calculated orbital solutions, shown for the primary (top) and the secondary (bottom), for data from 2007 (circles) and from 2008 (crosses).

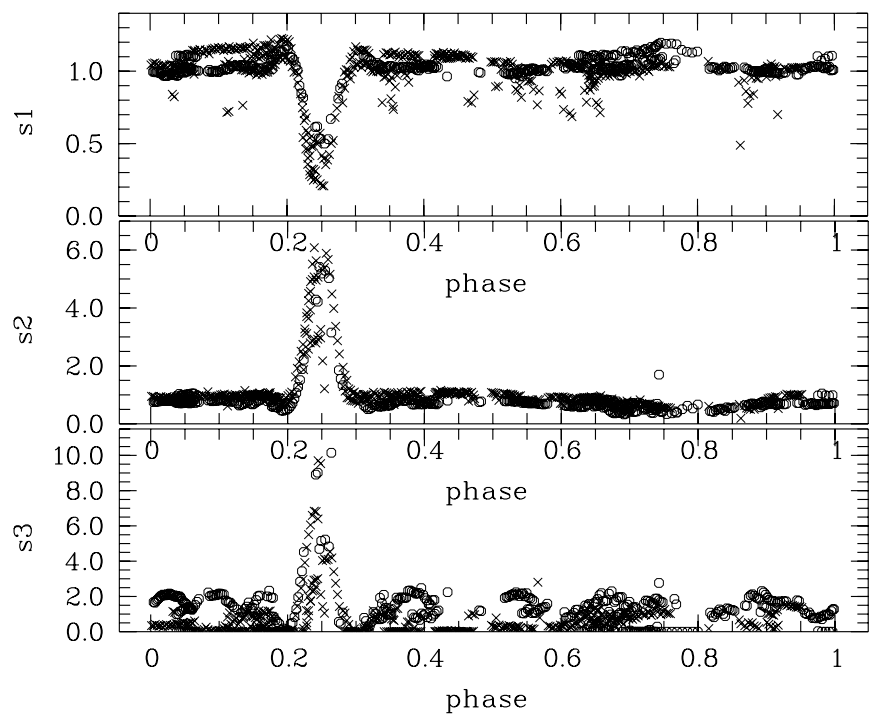

Figure 5. Same as Figure 3 but for the calculated line strengths, from top to bottom for primary, secondary, and third component, respectively.

pronounced Rossiter effect in the RVs of both stars during the corresponding eclipses.

M. Zejda et al. (2010, in preparation) derived photometric solutions using two different models and obtained for the orbital inclination the values of $86.74 \pm 0.03$ and $87.10 \pm 0.08$. In the following, we will use the weighted mean of $i=86.8 \pm 0.3$. Inserting the derived elements into

$$
\begin{aligned}
& M_{1}=\frac{P K_{1}^{3}(1+q)^{2}}{q^{3} \sin ^{3} i} \times 1.035793 \times 10^{-7} M_{\odot} \\
& M_{2}=q M_{1} \\
& a=\frac{P K_{1}(1+q)}{q \sin i} \times 0.0197714 R_{\odot},
\end{aligned}
$$

we get for the absolute masses and the separation of the TW Dra system: $M_{1}=(2.01 \pm 0.22) M_{\odot}, M_{2}=(0.89 \pm 0.07) M_{\odot}$, and $a=(12.10 \pm 0.47) R_{\odot}$.

Figure 5 shows the variation of line strengths with orbital phase computed with KOREL. The line strengths of the primary have a minimum during primary eclipse and a maximum during secondary eclipse. As expected, the line strengths of the secondary behave in the opposite way. The bottom panel of Figure 5 shows the more or less random variation of the line strength of the third component, depending on nightly seeing conditions and orientation of the spectrograph's slit. Only during 
primary eclipse where the light of the primary is dimmed by more then 2 mag can a pronounced maximum be seen. From the line strengths of the primary and secondary of TW Dra, we see no systematic differences between years 2007 and 2008 but variations on a short-term scale between different orbital revolutions. We cannot directly decide, however, if the shortterm variations are intrinsic or feigned by the varying influence of the third component.

\section{NORMALIZATION OF THE EXTRACTED SPECTRA}

Whereas the orbital elements have been determined using the full data set, it was not possible to separate the spectra of the three components in this way. The reason is that, due to the inconvenient time sampling, we always got a strong ripple (wavelike structure) in the continua. Only by restricting the calculation to the data obtained in 2007 did the resulting extracted spectra show sufficiently straight and smooth continua.

Since the individual continua of the KOREL-extracted spectra are a priori unknown, we have to renormalize the spectra. Let $r_{i}$ be the KOREL output spectra and $R_{i}$ the spectra normalized to the individual continua, and $l_{i}=1-r_{i}, L_{i}=1-R_{i}$ the corresponding line depths. The extracted spectra are normalized to the common continuum $C$ of all three stars:

$$
l_{i}=\alpha_{i} L_{i}, \quad \sum \alpha_{i}=1, \quad i=1, \ldots, 3,
$$

where $\alpha_{i}=C_{i} / C$ is the ratio between the continuum flux of component $i$ and the total continuum $C=\sum C_{i}$.

We want to compute intensities $R_{i}$ that are normalized to the individual continua $C_{i}$. This non-trivial task that is usually solved by assuming some flux ratio between the components approximated from photometry is complicated here by the fact that the third component's light contributes in a more or less random way to the spectra. We could solve the problem by using two advantageous facts: first, we have one spectrum that contains light only from the third component but not from the close binary. It was taken during primary eclipse under good seeing conditions by guiding the telescope on the third component. We will call the line intensities of this spectrum $R_{3}^{s}$. And second, the primary eclipse is a total one and spectra taken during Min I do not contain any light from the primary.

In the following, we will assume that the wavelength dependence of the continuum flux ratios along our wavelength region of interest can be neglected. Results will show that this approximation is justified. From this single spectrum of the third component, we find

$$
\alpha_{3}=\left\langle l_{3} / L_{3}^{s}\right\rangle,
$$

where the brackets mean the averaged mean. Concerning the uncertainty in the continuum levels in the KOREL and in the measured spectra, a more convenient way to normalize the KOREL spectra is to use a linear regression and $\sigma$-clipping according to

$$
L_{3}^{s}=a+b l_{3},
$$

where $b=1 / \alpha_{3}$ and $a$ corresponds to some continuum shift.

From our spectra we measured $\alpha_{3}=0.0840$. Figure 6 shows a comparison between the single observed spectrum of the third component and its normalized, extracted KOREL spectrum. The fit is almost perfect.

Now we know the normalized spectrum of the third component, i.e., $R_{3}$ and $L_{3}$. Spectra observed during the total primary eclipses do not contain any contributions from the primary and we have

$$
r^{\mathrm{Min} \mathrm{I}}=\frac{R_{2} C_{2}+R_{3} C_{3}^{\mathrm{Min} \mathrm{I}}}{C_{2}+C_{3}^{\operatorname{Min} \mathrm{I}}} .
$$

Introducing the ratio of the continuum fluxes between the third component in the spectra during primary minimum (note that this contribution varies in a random way) and the continuum flux of the secondary $\alpha_{3}^{\prime}=C_{3}^{\operatorname{Min} \mathrm{I}} / C_{2}$, we get

$$
R_{2}=\left(1+\alpha_{3}^{\prime}\right) r^{\mathrm{MinI}}-\alpha_{3}^{\prime} R_{3} .
$$

This can be transformed by using Equation (2) to

$$
l^{\mathrm{Min} \mathrm{I}}=\frac{l_{2}}{\alpha_{2}}+\alpha_{3}^{\prime}\left(L_{3}-l^{\operatorname{Min} \mathrm{I}}\right) .
$$

We used Equation (7) to obtain the continuum flux ratios by a least-squares fit between the spectrum $l^{\operatorname{Min} I}$ observed during primary minimum, the KOREL-extracted spectrum of the secondary $l_{2}$, and the already renormalized spectrum of the third component $L_{3}$ and yield $\alpha_{2}=0.100, \alpha_{3}^{\prime}=0.283$.

Figure 7 compares the spectrum during primary minimum (black) and the best fit according to Equation (7) (green) obtained from

$$
r^{\mathrm{MinI}}=\frac{1+\alpha_{3}^{\prime} R_{3}-\left(1-r_{2}\right) / \alpha_{2}}{1+\alpha_{3}^{\prime}} .
$$

To check for the influence of the third component during primary eclipse, we additionally computed the spectrum shown in Figure 7 in red color by setting $R_{3}$ to unity. The influence of the third component on the line profiles can be clearly seen from the difference between the almost perfect fit by the green spectrum and the fit by the red one. From the goodness of fit, we conclude that it is not necessary to account for the wavelength dependence of the flux ratios within the given wavelength interval. The fact that the fit is almost perfect proves, together with the results shown in Figure 6, that KOREL performs the separation of spectra very well and that our approach to the problem of renormalization gives reliable results.

Now we know $\alpha_{2}$ and $\alpha_{3}$, from which we get $\alpha_{1}=0.816$. The continuum flux ratios with regard to the primary follow to $C_{2} / C_{1}=0.123, C_{3} / C_{1}=0.103$, where the latter value is the mean ratio during observations on/off the slit and does not give the real flux ratio between the stars.

\section{SPECTRUM ANALYSIS}

We used the normalized, extracted spectrum of the primary to determine its atmospheric parameters and elemental abundances. For the calculation of model atmospheres, we used the program LLmodels (Shulyak et al. 2004) and for the computation of synthetic spectra the SynthV code (Tsymbal 1996). Parameters have been obtained in an iterative procedure. We started with the computation of model atmospheres on a grid in $T_{\text {eff }}(7500-8500 \mathrm{~K}$, steps of $100 \mathrm{~K})$ and $\log g$ (3.6-4.1, steps of 0.1 ). These first models were based on solar abundances and a micro-turbulence $\xi$ of $2 \mathrm{~km} \mathrm{~s}^{-1}$. From each model atmosphere, we computed synthetic spectra with SynthV in the range from $4890 \AA$ to $5670 \AA$ for different $v \sin i$ and compared them with the observed spectrum applying the $\chi^{2}$ criterion. In this way, we obtained first optimized values of $T_{\text {eff }}$ and $\log g$. In the next step, we varied the micro-turbulence and all elemental abundances for which we found a contribution in the observed spectral range 

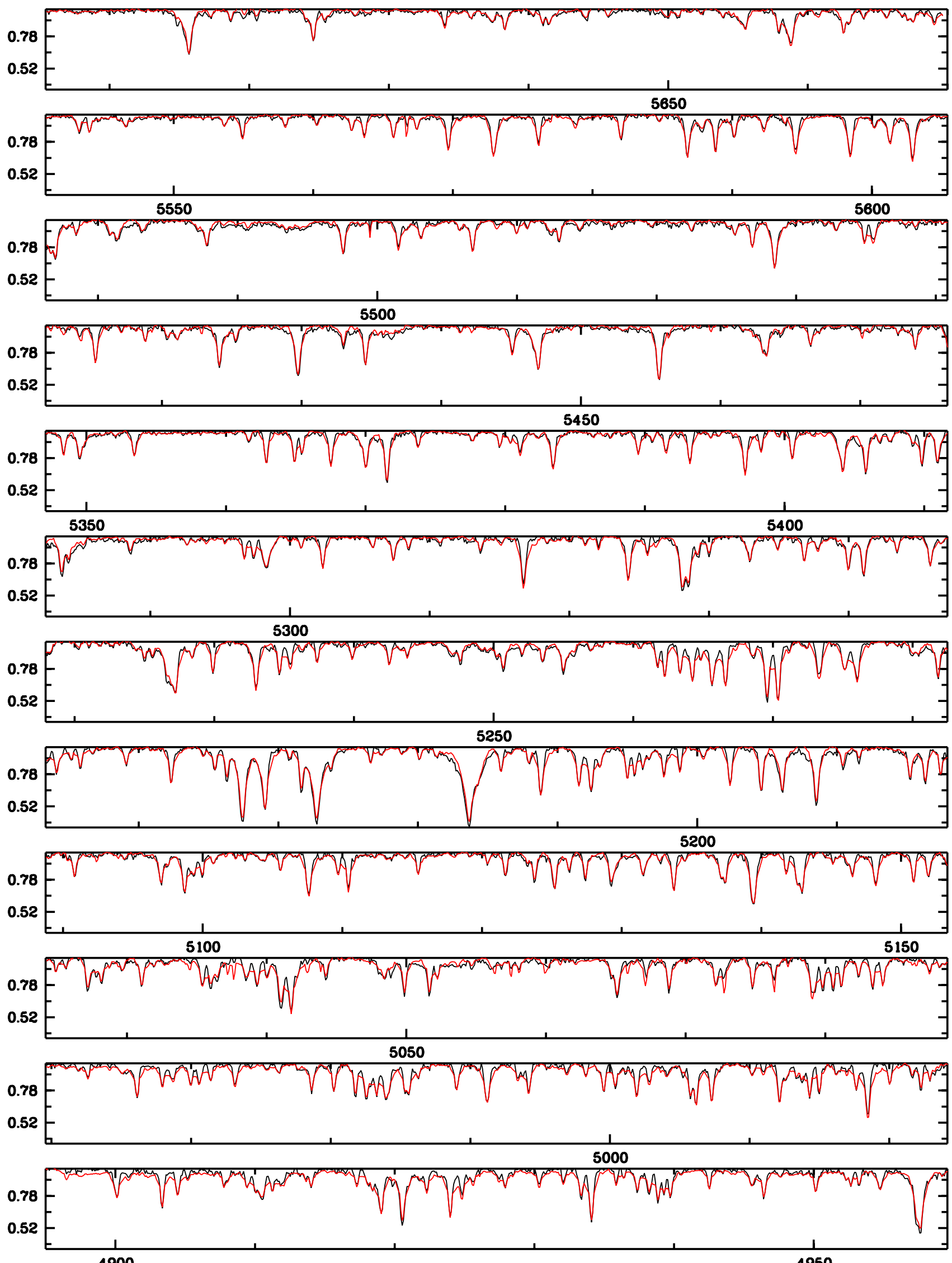

Figure 6. Sharp-lined, third component's spectrum: thick line (black in online version) is the single observed spectrum, thin line (colored red in online version) is the normalized extracted spectrum.

(A color version of this figure is available in the online journal.) 

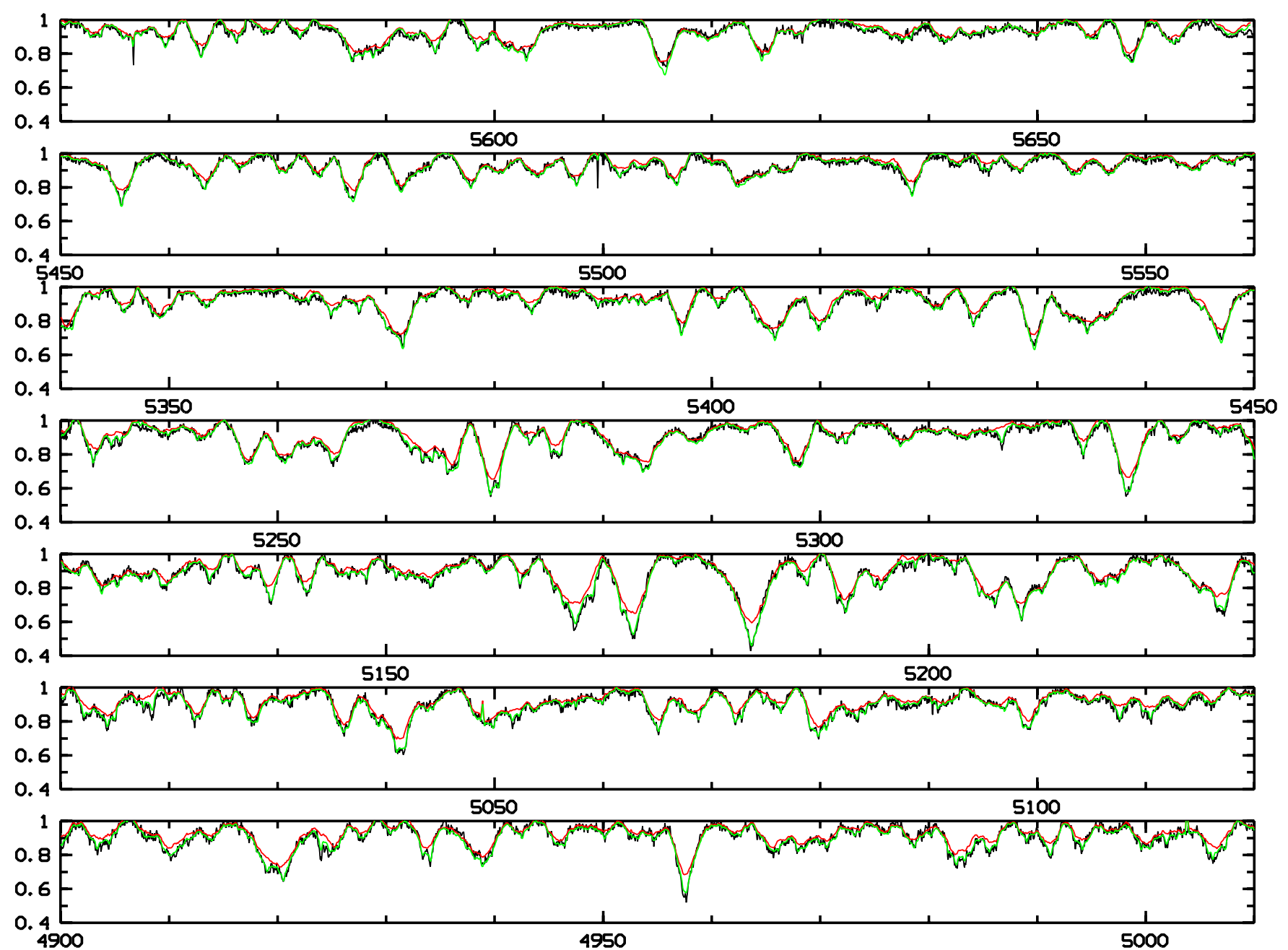

Figure 7. Fit of the observed spectrum during Min I (black) by the extracted spectrum of the secondary alone (red) and by adding the spectrum of the third component (green).

(A color version of this figure is available in the online journal.)

using SynthV to derive their optimum values. These have been finally given as new input parameters to LLmodels to compute new model atmospheres, again on a grid in $T_{\text {eff }}$ and $\log g$.

The described procedure was repeated four times where we refined the grid using smaller ranges and smaller step widths ( $\Delta T_{\text {eff }}=25 \mathrm{~K}, \Delta \log g=0.02$ in the last step). No changes in the $\chi^{2}$ of the optimum solution could be detected after the third iteration. In this way, we finally obtained the best parameters based on a consistent model atmosphere. Realistic errors of the parameters can only be obtained by computing synthetic spectra on a multidimensional grid of all parameters including the elemental abundances. Since we had not the computer power to do this, we computed the error of each single parameter from the $\chi^{2}$ statistics based on a one-dimensional grid centered at minimum $\chi^{2}$ by fixing all other parameters to their optimum values. The errors obtained in this way are only rough estimations; the true errors can be slightly larger.

We obtained $T_{\text {eff }}=(8150 \pm 20) \mathrm{K}, \log g=3.88 \pm 0.02$, $v \sin i=(47.1 \pm 0.5) \mathrm{km} \mathrm{s}^{-1}$, and $\xi=(2.9 \pm 0.3) \mathrm{km} \mathrm{s}^{-1}$. Table 3 lists the derived abundances, the error was estimated to \pm 0.03 dex for $\mathrm{Fe}$ and \pm 0.05 dex for the other elements. The abundances are close to solar ones. Slight overabundance was found for $\mathrm{Mg}, \mathrm{Sc}, \mathrm{Cr}, \mathrm{Fe}$, and $\mathrm{Ni}$ with $0.35,0.26,0.20,0.16$, and 0.20 dex, respectively, while $\mathrm{O}(-0.14 \mathrm{dex})$ and $\mathrm{Si}(-0.13 \mathrm{dex})$
Table 3

Elemental Abundances of the Primary of TW Dra

\begin{tabular}{lccc}
\hline \hline \multicolumn{1}{c}{$\mathrm{C}$} & $\mathrm{O}$ & $\mathrm{Mg}$ & $\mathrm{Si}$ \\
\hline$-3.56(+0.09)$ & $-3.56(-0.14)$ & $-4.16(+0.35)$ & $-4.66(-0.13)$ \\
$\mathrm{Ca}$ & $\mathrm{Sc}$ & $\mathrm{Ti}$ & $\mathrm{Cr}$ \\
$-5.63(+0.10)$ & $-8.73(+0.26)$ & $-7.07(+0.07)$ & $-6.20(+0.20)$ \\
$\mathrm{Fe}$ & $\mathrm{Ni}$ & $\mathrm{Y}$ & \\
$-4.43(+0.16)$ & $-5.61(+0.20)$ & $-9.74(+0.09)$ & \\
\hline
\end{tabular}

Note. Values in parentheses give the deviation from standard solar abundance.

are slightly underabundant. Figure 8 shows the best fit of the renormalized, extracted spectrum of the primary based on the derived abundances and atmospheric parameters. We conclude that the primary of the Algol-type system is a normal A-type star.

\section{CALCULATION OF THE COMPOSITE SYNTHETIC SPECTRA}

We used the Shellspec07_inverse program for the computation of synthetic, composite spectra of TW Dra at arbitrary orbital phases. The program is based on the Fortran 77 code "Shellspec07" written by Budaj \& Richards (2004, see also 


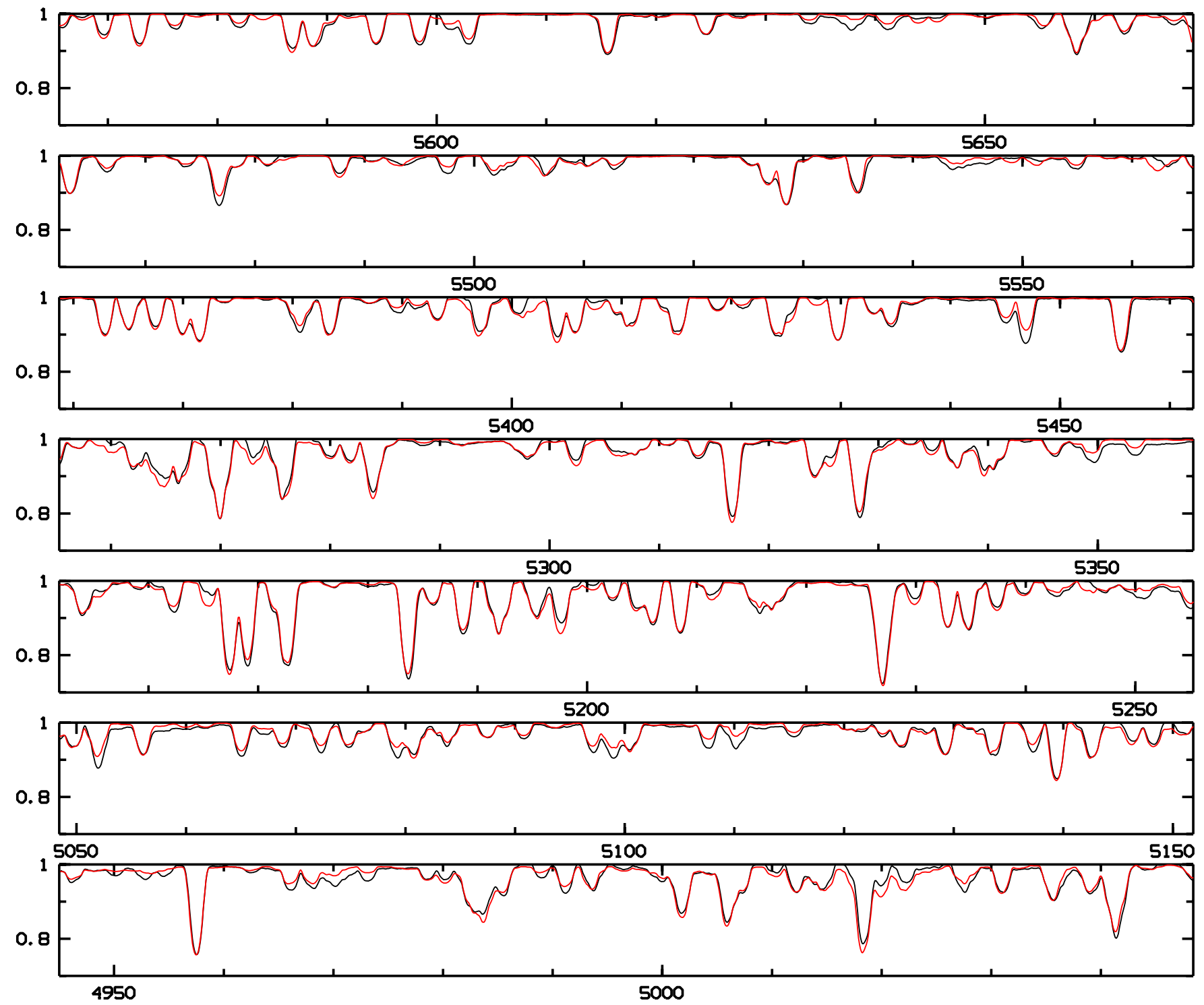

Figure 8. Best fit (thin line in printed version and colored red in online version) of the extracted spectrum of the primary (thick line in printed version and black in online version).

(A color version of this figure is available in the online journal.)

Budaj et al. 2005) and is specially designed for the fine-tuning of stellar and system parameters of eclipsing binaries based on spectroscopic data. The program solves the inverse problem by means of the Levenberg-Marquardt nonlinear optimization algorithm (Marquardt 1963). The code provides an improved calculation of the effects of limb and gravity darkening as well as an accurate normalization of the computed spectra to the local continuum. It is described in more detail in Tkachenko et al. (2009).

\subsection{Application to the TW Dra System}

The existence of the third, visual component, as can be strikingly seen from Figure 7, complicates the modeling of the close binary system. To count for the third light, we implemented an additional subroutine into Shellspec07_inverse. It solves for the problem by means of a least-squares fit in the sense of

$$
\left(R_{\mathrm{obs}}-\alpha_{1} R_{c}-\alpha_{2} R_{3}\right)^{2} \rightarrow \min ,
$$

where $R_{\text {obs }}$ is the observed composite spectrum that includes some random amount of light from the third component, $R_{c}$ is the spectrum computed by Shellspec07_inverse at the corresponding orbital phase, and $R_{3}$ is the normalized observed spectrum of the third component. The free parameters $\alpha_{1}$ and $\alpha_{2}$ represent two physical parameters: the ratio of the continuum flux of the third light in the spectrum to the continuum flux of the Algol-type system, i.e., $C_{3} /\left(C_{1}+C_{2}\right)=\alpha_{2} / \alpha_{1}$, and a correction factor for a possibly inaccurate continuum normalization $\beta=\left(\alpha_{1}+\alpha_{2}\right)^{-1}$.

We used the LLmodels code (Shulyak et al. 2004) for the calculation of atmosphere models for the more massive hot primary component and MARCS models (Gustafsson et al. 2008) for the cool secondary. In the case of the secondary, the molecular line list by Kurucz (1995) has been used, additionally to the list of atomic lines taken from the VALD database (Kupka et al. 2000). Intrinsic line profiles have been calculated with the SynthV program (Tsymbal 1996) on a grid of different temperatures and nine different angles $\Theta$ between the line of 

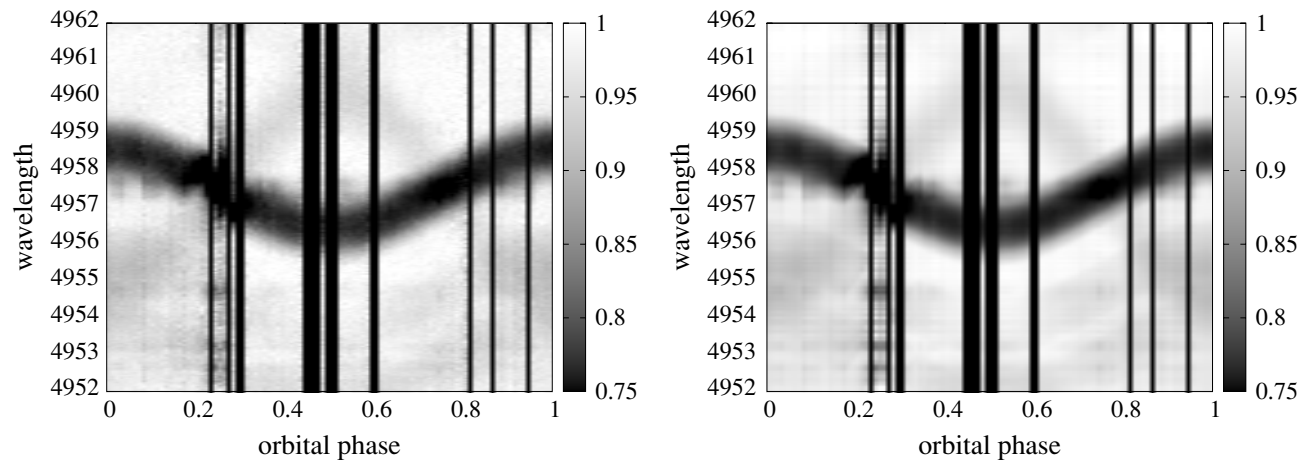

Figure 9. Fe I $4957 \AA$ A line profiles observed in 2007 (left) and calculated with Shellspec07_inverse (right), folded with the orbital period. The phase of primary minimum was shifted to 0.25 for better visualization. The phase of largest separation in this case corresponds to 0.0 or 0.5 .
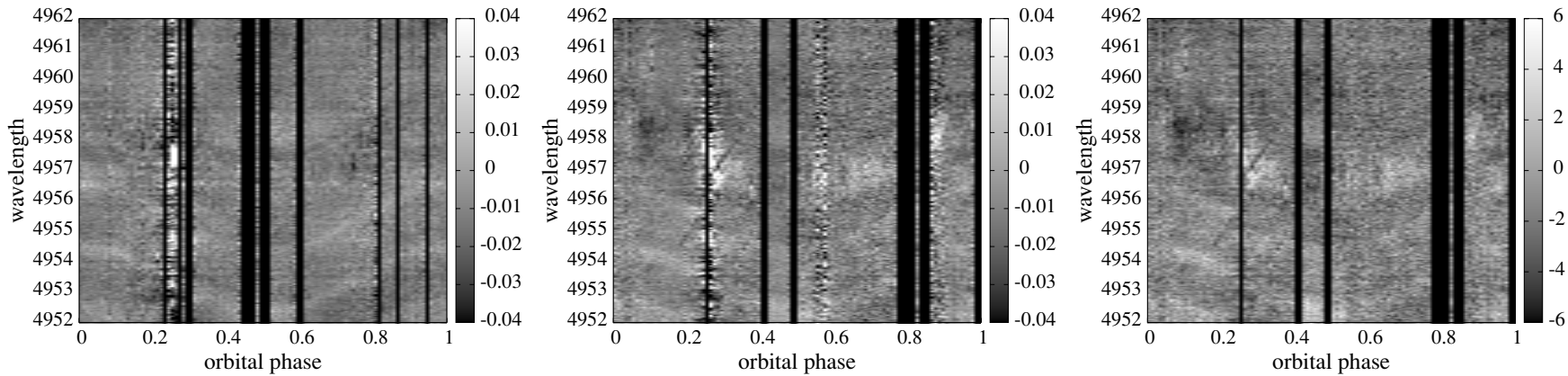

Figure 10. $O-C$ line intensity residuals obtained from the Fe I $4957 \AA$ line observed in 2007 (left), in 2008 (center), and the $\chi^{2}$-distribution corresponding to the 2008 residuals (right). Orbital phases are the same as in Figure 9.

sight and the normal of the stellar surface and provided to Shellspec07_inverse.

In the Shellspec07_inverse calculations, we used the elemental abundances and $\log g$ of both stars as derived from the analysis of the extracted spectra and determined the following free parameters: effective temperatures $T_{\mathrm{eff}_{1,2}}$ and $\mathrm{RV}$ semi-amplitudes $K_{1,2}$ of both components, $v_{1} \sin i$ of the primary, systemic $\gamma$-velocity, radius of the primary $R_{1}$, and orbital inclination $i$. For the cool secondary, we assumed synchronous rotation and Roche-lobe filling geometry; the deviation of the shape of the primary from sphericity was neglected. For the analysis we used mean profiles built from the four $\mathrm{Fe}$ I lines at 4958, 5056, 5616, and $5625 \AA$ and averaged them into 100 orbital phase bins calculated from $P=2.8068491$ days and Min I $=2454400.97997$ as given in Section 4.

Figure 9 shows an example of line profiles observed in 2007 together with those computed with Shellspec07_inverse. Each column corresponds to a single composite line profile. Vertical dark stripes indicate observational gaps. The phase of primary minimum was shifted to 0.25 for better visualization. At this phase, strong distortions of the line of the primary due to the Rossiter effect can be seen.

Figure 10 shows in the first two panels the $O-C$ line intensity residuals of the Shellspec07_inverse calculations folded with orbital period. Largest values are obtained at orbital phases where the observed spectra have the lowest signal-to-noise ratio due to the faintness of the object during primary eclipse and/or due to a smaller number of averaged spectra falling into the corresponding bin. To suppress the influence of this effect, we did not use the rms of the $O-C$ values as a measure for the goodness of fit but the $\chi^{2}$ value of the fit which we define as the $O-C$ values normalized to the individual errors of measure- ment. The resulting, much smoother distribution of these values can be seen from the right panel of Figure 10.

The finally derived stellar and system parameters are listed in the last row of Table 4 which will be used in the following section for a comparison with previous determinations. Figure 11 compares selected calculated line profiles with the observed ones. Each profile is shifted by a constant value for better visualization, orbital phases are given to the right. As can be seen from Figures 10 and 11, our model matches the observations very well and there are no obvious differences between the results obtained from the data from 2007 and from 2008. The resulting mean value of $\chi^{2}$ is 1.47 for the spectra from 2007 and 1.77 for those from 2008 . Only during primary eclipse can a small bright region be seen in the $O-C$ distributions where the computed line strengths are stronger than the observed ones. Figure 12 allows for a closer look at this problem. First, one notes the drastic difference between the observed spectrum (filled circles) and the spectrum computed by Shellspec07_inverse without including the third component (dashed line). It comes mainly from the fact that during the total primary eclipse TW Dra is fainter by $2 \mathrm{mag}$ and the light contributions from the secondary and the third component are of the same order, and maybe partly from an inaccurate normalization of the observed latetype spectrum at this phase. Normally, both problems should have been solved by including the observed spectrum of the third component (dash-dotted line in Figure 12) into the calculations by means of the least-squares fit as described before. It can be seen from Figure 12 (solid profile) that this is true for the continuum and the wings of the computed line profile but not for the line center where the computed line is too strong. The structure of the overestimated part of the computed profile resembles the line core from the third component and it seems 


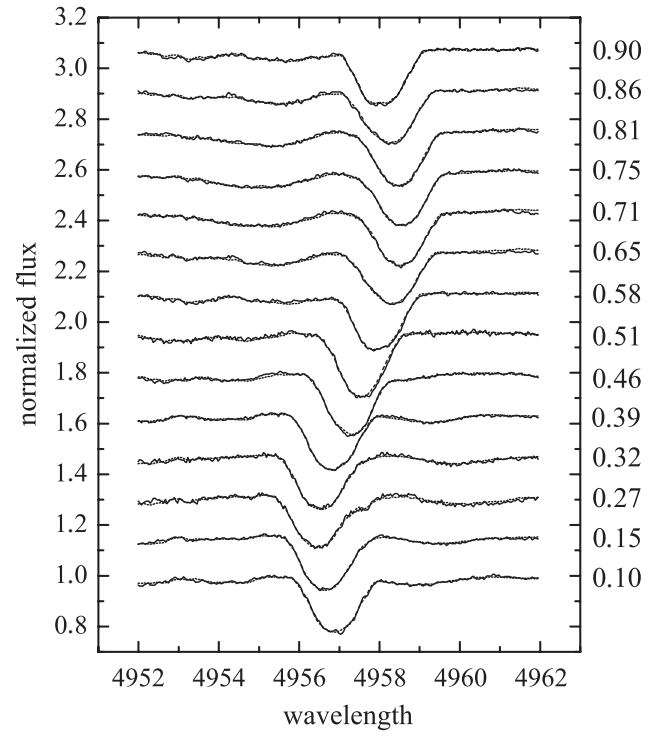

Figure 11. Comparison of calculated (dashed) and observed (solid) composite line profiles of TW Dra. The orbital phase is indicated to the right. Phase 0.0 corresponds to the primary minimum.

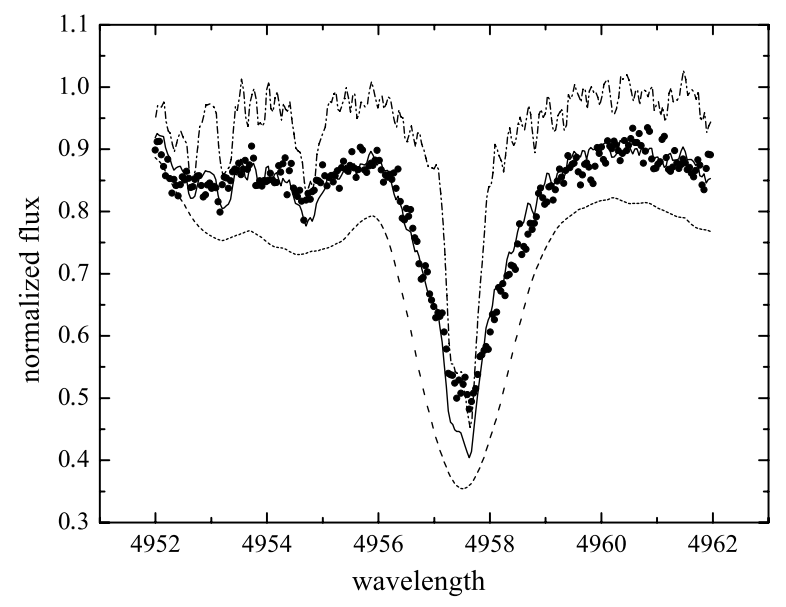

Figure 12. Composite line profiles observed during primary eclipse (big dots), calculated with Shellspec07_inverse including all three components (solid), calculated without the third component (dashed), and observed profile of the third component (dash-dotted).

that for some reason our fitting procedure overestimates the light contribution from the third component in this special case.

\section{DISCUSSION}

Based on all spectra taken in 2007 and 2008 we derived the orbital solution by means of the KOREL program. It was not possible to separate the spectra of the individual components using the full data set, however, because wavelike structures appeared in the computed continua of the extracted spectra. We assume that the time sampling of the spectra including a large gap between the two epochs of observations caused this behavior. Only by restricting the calculations to the data from 2007 were we able to obtain sufficiently straight and smooth continua as was shown in Section 5.

The almost perfect fit obtained for the observed spectrum of the third (visual) component shows that (1) the KOREL program delivers reliable results with respect to separating the composite into individual spectra even in our very special case of three components where the contribution of one component

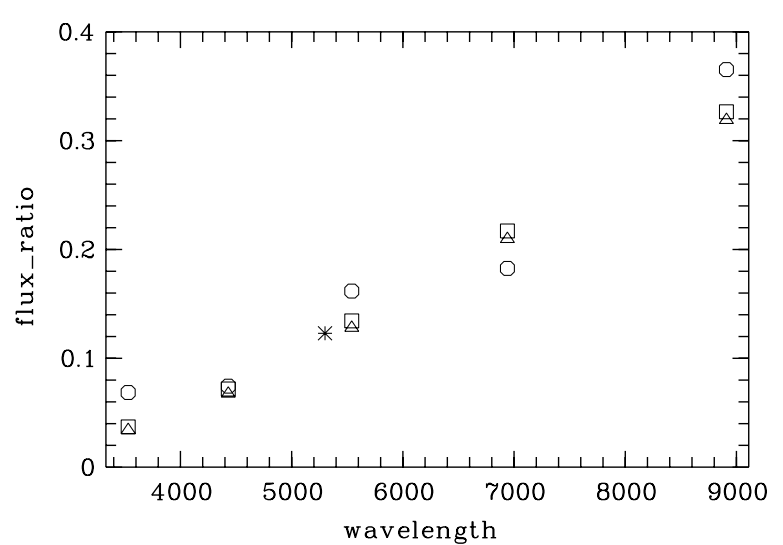

Figure 13. Flux ratios secondary/primary obtained by M. Zejda et al. (2010, in preparation) from $U B V R I$ photometry using FOTEL (circles), PHOEBE (squares), and our value (asterisk; see the text).

shows non-predictable changes, (2) there was no need to account for the wavelength dependence of the continuum flux ratios within the region of interest, and (3) that our approach to renormalize the spectra works well.

In the framework of this renormalization, we obtained the specific flux ratio between secondary and primary of 0.123 , valid for a central wavelength of $5300 \AA$. In Figure 13, we show the ratio of fractional fluxes derived by M. Zejda et al. (2010, in preparation) from $U B V R I$ photometry using different methods like FOTEL (Hadrava 2004a, shown by circles), PHOEBE (Prša \& Zwitter 2005) using the separate (shown by squares), and the Algol-type (triangles) option. We have plotted the flux ratio versus the wavelength derived from the center of gravity of the corresponding passband. Our value derived during the normalization of the KOREL extracted spectra is shown by the asterisk, and it can be seen that it fits well into the diagram.

The Shellspec07_inverse code has been used to optimize the stellar and system parameters of TW Dra. The existence of the third, visual component in the system strongly complicated the modeling of the close binary, however. Table 4 collects all the parameters derived by us in the present work together with those known from the literature so far. Errors of measurement (if known) are given in units of the last digit in parentheses. The effective temperature of the primary of $8160 \mathrm{~K}$ derived with Shellspec07_inverse is consistent within the errors of measurement with that obtained from the analysis of the extracted spectrum. It is also in good agreement with the value of $8180 \mathrm{~K}$ found by M. Zejda et al. (2010, in preparation). For the cool secondary we derive an effective temperature of $4540 \mathrm{~K}$ which is about $100 \mathrm{~K}$ higher than that given by M. Zejda et al. (2010, in preparation) and Giuricin et al. (1980). Because the SynthV program used to compute the synthetic spectra does not consider the effects of non-sphericity and gravity darkening, we did not estimate the temperature of the companion from its extracted spectrum but determined it only with Shellspec07_inverse.

With Shellspec07_inverse we derived the radius of the primary to $2.58 R_{\odot}$ which is in good agreement with the values of $2.53 R_{\odot}$ and $2.50 R_{\odot}$ found by M. Zejda et al. (2010, in preparation) and Al-Naimiy \& Al-Sikab (1984), respectively. For the orbital inclination we derive $86^{\circ} 8$, a value lying between the values given by M. Zejda et al. (2010, in preparation) and Giuricin et al. (1980). Whereas the scatter in the values of the separation of the system derived by different authors is small, the large scatter in mass ratios is obvious. On the other hand, 
Table 4

TW Dra: Compilation of Stellar Parameters From the Literature

\begin{tabular}{|c|c|c|c|c|c|c|c|c|c|c|}
\hline Reference & $\begin{array}{c}T_{1} \\
(\mathrm{~K})\end{array}$ & $\begin{array}{c}T_{2} \\
(\mathrm{~K}) \\
\end{array}$ & $\log g_{1}$ & $\log g_{2}$ & $\begin{array}{c}R_{1} \\
\left(R_{\odot}\right)\end{array}$ & $\begin{array}{c}M_{1} \\
\left(M_{\odot}\right)\end{array}$ & $\begin{array}{c}M_{2} \\
\left(M_{\odot}\right)\end{array}$ & $q$ & $\begin{array}{c}a \\
\left(R_{\odot}\right)\end{array}$ & $\begin{array}{c}i \\
(\operatorname{deg}) \\
\end{array}$ \\
\hline A & & & & & 2.4 & 1.9 & 0.82 & 0.43 & 12 & \\
\hline B & & & & & & 1.7 & 0.8 & 0.47 & & \\
\hline $\mathrm{C}$ & 8060 & 4450 & & & 2.4 & & & 0.47 & & $86.1(2)$ \\
\hline $\mathrm{D}$ & & & & & 2.5 & & & & 12 & \\
\hline $\mathrm{E}$ & 8180 & 4407 & 3.96 & 3.23 & $2.53(3)$ & $2.11(5)$ & $0.85(2)$ & $0.403(2)$ & $12.0124(3)$ & $87.13(3)$ \\
\hline
\end{tabular}

Notes. Sources are: (A) Kopal \& Shapley 1956; (B) Popper 1978; (C) Giuricin et al. 1980; (D) Al-Naimiy \& Al-Sikab 1984; (E) M. Zejda et al. 2010, in preparation; $(\mathrm{F})$ This paper.

we see that the values of the absolute masses and of the mass ratio determined in the two most recent investigations (M. Zejda et al. 2010, in preparation, and this work) agree very well within the errors of measurement.

The values of the RV semi-amplitudes $K_{1}=(64.0 \pm$ $0.02) \mathrm{km} \mathrm{s}^{-1}, K_{2}=156 \pm 1 \mathrm{~km} \mathrm{~s}^{-1}$, projected rotational velocity of the primary $v \sin i=(49.9 \pm 0.2) \mathrm{km} \mathrm{s}^{-1}$, and systemic velocity $\gamma=(-0.8 \pm 0.1) \mathrm{km} \mathrm{s}^{-1}$ derived with Shellspec07_inverse are consistent with those obtained from the KOREL orbital solution and from the analysis of the extracted spectrum of the primary. From the derived radius of the primary and its $v \sin i$ we obtain a rotation period of $P_{\text {rot }}=(2.62 \pm$ 0.03 ) days. Thus the primary rotates synchronously with a factor of $(1.07 \pm 0.01)$ compared to the orbital period. For the secondary, we assumed synchronized rotation and a Roche-lobe filling shape. For this shape, we determined in the equatorial plane the three radii $R_{\text {point }}=5.00 R_{\odot}, R_{\text {back }}=4.02 R_{\odot}$, and $R_{\text {side }}=3.62 R_{\odot}$ so that the local $v \sin i$ varies between 65 and $90 \mathrm{~km} \mathrm{~s}^{-1}$. $R_{\text {pole }}$ was determined to $3.47 R_{\odot}$, for the effective radius of the secondary that approximates the spherical case we estimate $3.7 R_{\odot}$.

We compared the obtained stellar parameters with standard values given by Schmidt-Kaler (1982) and used the tables by de Jager \& Nieuwenhuijzen (1987) for the temperature calibration to deduce spectral types and evolutionary states. The derived temperature of the primary corresponds to spectral type A5 for a mean sequence or slightly evolved star. The derived mass is in agreement with this assumption. According to the obtained radius and $\log g$ the primary is slightly evolved. According to the derived mass, radius, and $\log g$, the secondary is a subgiant. For LC IV, the derived temperature gives spectral type K 1 .

The line profile fit shown in Figure 11 and the $\mathrm{O}-\mathrm{C}$ value distributions shown in Figure 10 are based on the parameters as listed in the last row of Table 4. From both figures it can be seen that our model matches the observations very well. Only during primary eclipse can a difference between the model and observations be seen (Figure 12), as already discussed in the previous section. We checked for two possible explanations of the observed effect: first, the problem could be solved if there would exist some additional light source acting only during primary eclipse. But any hot spot of arbitrary size on the backsurface of the secondary pointing toward the observer (away from the primary) or other source of such additional light could be seen from a large range in orbital phase around primary minimum. The problem is that we can fit the observed profiles perfectly without such an assumption in all orbital phases except for the primary minimum itself. Second, some of the derived system parameters could be wrong. There are three parameters that influence the line shape in particular during primary minimum: the radius of the primary $R_{1}$, the orbital inclination $i$, and the inclination $\phi$ of the rotation axis in the tangential plane of the observer ( $\phi$ is measured in a plane perpendicular to those of $i$ ). It is possible to reproduce the line profiles observed during primary eclipse by adjusting one of the first two parameters accordingly. But in both cases we end up with a partial eclipse instead of a total one. And in the case of a changed orbital inclination, the fit in the out-of-eclipse phases is worse due to the fact that it changes the mass of the primary and the separation between the two components. The third parameter is also out of the question because any deviation of the rotation axis from $90^{\circ}$ causes asymmetric line profiles. Thus we cannot give an explanation for the overfitting of the line profiles during primary eclipse.

There are no obvious differences between the results obtained from the spectra taken in 2007 and in 2008. The resulting mean value of $\chi^{2}$ is 1.47 for the spectra from 2007 and 1.77 for those from 2008.

\section{CONCLUSIONS}

Our application of the KOREL program showed, maybe for the first time directly, that KOREL delivers reliable extracted spectra of a spectroscopic triple system. From the analysis of the extracted spectrum of the primary we conclude that it is a normal A-type star with abundances that are about solar.

The derived orbital solution and the stellar parameters obtained from the analysis of the extracted spectrum of the primary have been used as starting values for fine-tuning with the Shellspec07_inverse program. Using Shellspec07_inverse we obtained optimized stellar and system parameters of both components of the Algol-type system considering the non-spherical shape of the Roche-lobe filling cool secondary and gravity darkening.

Results show, that TW Dra can be modeled in almost all orbital phases without taking any effects of mass transfer like circumbinary matter, accretion disk, or gas stream into account. We found no obvious differences between the two epochs of observation in 2007 and 2008 so we conclude that the star was in a non-active phase during both epochs.

All stellar parameters derived here spectroscopically agree very well with those from the most recent photometric investigation (M. Zejda et al. 2010, in preparation) using the WilsonDevinney program. In cases of the radius of the primary and the orbital inclination, we reach the same accuracy as obtained from photometry. For the masses and the mass ratio, the derived errors are about twice the photometric errors. According to the obtained stellar parameters, the primary of RZ Cas is a slightly evolved A5 V star and the secondary a K1 IV subgiant. 
In our previous, first application of Shellspec07_inverse to the Algol-type star RZ Cas (Tkachenko et al. 2009), we could model the system in all orbital phases including the primary eclipse. We assume that the overfitting of the line profiles of TW Dra during primary minimum is due to the complications introduced by the light of the third, visual component but cannot give any more detailed explanation. This problem should be solved with a further observing campaign when we will yield spectra obtained under better seeing conditions without any contributions from the visual companion.

We thank Jan Budaj for providing us with the latest version of the Shellspec07 program and for his help in software usage. We are grateful to Vadim Tsymbal for providing us with the SynthV code and to Oleg Khochukov for the modified version of the Levenberg-Marquardt algorithm as well as for their steady help and useful comments.

Facilities: TLS, BOAO:1.8m

\section{REFERENCES}

Al-Naimiy, H. M. K., \& Al-Sikab, A. O. 1984, Ap\&SS, 103, 115

Baglow, R. L. 1952, Publ. David Dunlap Obs. 2

Budaj, J., \& Richards, M. T. 2004, Contrib. Astron. Obs. Skalnate Pleso, 34, 167

Budaj, J., Richards, M. T., \& Miller, B. 2005, ApJ, 623, 411

de Jager, C., \& Nieuwenhuijzen, H. 1987, A\&A, 177, 217

Giuricin, G., Mardirossian, F., \& Fredolin, F. 1980, Ap\&SS, 73, 389
Gustafsson, B., Edvardsson, B., Eriksson, K., Jørgensen, U. G., Nordlund, A., \& Plez, B. 2008, A\&A, 486, 951

Hadrava, P. 2004a, Publ. Astron. Inst. ASCR, 92, 1

Hadrava, P. 2004b, Publ. Astron. Inst. ASCR, 92, 15

Kim, S.-L., Lee, J. W., Kwon, S.-G., Youn, J.-H., Mkrtichian, D. E., \& Kim, C. 2003, A\&A, 405, 231

Kopal, Z., \& Shapley, M. B. 1956, Jodrell Bank Ann., 1, 141

Kupka, F., Ryabchikova, T. A., Piskunov, N. E., Stempels, H. C., \& Weiss, W. W. 2000, Baltic Astron., 9, 590

Kurucz, R. L. 1995, in ASP Conf. Ser. 78, Astrophysical Applications of Powerful New Databases, ed. S. J. Adelman \& W. L. Wiese (San Francisco, CA: ASP), 205

Kusakin, A. V., Mkrtichian, D. E., \& Gamarova, A. Yu. 2001, IBVS, 5106, 1

Lehmann, H., Tkachenko, A., \& Mkrtichian, D. E. 2009, Commun. Asteroseismol., 159, 45

Lehmann, H., Tkachenko, A., Tsymbal, V., \& Mkrtichian, D. E. 2008, Commun. Asteroseismol., 157, 332

Marquardt, D. 1963, SIAM J. Appl. Math., 11, 431

Popper, D. M. 1978, BAAS, 10, 608

Popper, D. M. 1989, ApJS, 71, 595

Prša, A., \& Zwitter, T. 2005, ApJ, 628, 426

Qian, S. B., \& Boonrucksar, S. 2002, New Astron., 7, 435

Schmidt-Kaler, T. 1982, in Landolt-Börnstein-Group VI Astronomy and Astrophysics, Vol. 2b, Stars and Star Clusters, ed. K. Schaifers \& H. H. Voigt (Berlin: Springer)

Shulyak, D., Tsymbal, V., Ryabchikova, T., Stütz, Ch., \& Weiss, W. W. 2004, A\&A, 428, 993

Tkachenko, A., Lehmann, H., \& Mkrtichian, D. E. 2009, A\&A, 504, 991

Tsymbal, V. 1996, in ASP Conf. Ser. 108, M.A.S.S.: Model Atmospheres and Spectrum Synthesis, ed. S. J. Adelman, F. Kupka, \& W. W. Weiss (San Francisco, CA: ASP), 198

Walter, K. 1978, A\&AS, 32, 57

Zejda, M., Mikulášek, Z., \& Wolf, M. 2008, A\&A, 489, 321

Zejda, M., Mikulášek, Z., Wolf, M., \& Pejcha, O. 2006, Ap\&SS, 304, 161 